\title{
Low-density open-cell foams in the NiTi system
}

\author{
David S. Grummon ${ }^{\text {a) }}$ \\ Department of Chemical Engineering and Materials Science, Michigan State University, East Lansing, \\ Michigan 48824 \\ John A. Shaw and Antoine Gremillet \\ Department of Aerospace Engineering, The University of Michigan, Ann Arbor Michigan 48109
}

(Received 15 July 2002; accepted 24 February 2003)

\begin{abstract}
It is shown that open-cell metallic foams having very low density, and that display martensite transformations required for shape memory and superelastic behavior, can be fabricated using a powder-metallurgy technique. Results are presented on experiments in which a polymeric precursor foam was coated with an equiatomic NiTi powder slurry and subsequently sintered to yield foams with relative densities as low as 0.039 . Although contaminated with interstitial impurities, they displayed unambiguous calorimetric signature of the $\mathrm{B} 2 \rightarrow \mathrm{B} 19^{\prime}$ transformation. The results are of considerable significance to potential applications requiring ultralightweight structures with the unusual dissipative and strain-recovery properties of NiTi shape-memory materials. (C) 2003
\end{abstract} American Institute of Physics. [DOI: 10.1063/1.1569036]

Shape memory and superelastic alloys based on equiatomic titanium-nickel are of technological and economic importance for a variety of applications. ${ }^{1,2}$ They display a unique inelastic strain recovery effect made possible by a reversible first-order athermal displacive transformation between highly ordered phases with widely differing crystallographic symmetry. In the NiTi system, the low-temperature monoclinic allotrope (the "martensite" phase) is easily deformed by twinning reactions. Strong long-range ordering imposes crystallographic constraints that allow recovery of strains as high as 0.08 to 0.10 on reversion to the hightemperature cubic phase (the "austenite"). The austenite-tomartensite transformation can also be induced by externally applied stresses, and subsequent isothermal reversion to the austenite on unloading gives rise to the transformational superelastic effect. Both shape memory and superelastic strain recovery offer the potential for an intrinsic "self-repairing" functionality. ${ }^{3}$ Furthermore, the highly dissipative characteristic of the transformational hysteresis is attractive for potential application to armor and vibration or to acoustic isolation problems. If they can be deployed as very-low-density metallic foams, ${ }^{4} \mathrm{NiTi}$ (or nitinol) shape-memory and superelastic materials (which are corrosion-resistant and biocompatible) may enable a variety of applications.

It is not surprising, then, that such materials have been eagerly pursued. Itin et $a .^{5}$ provide an excellent review of early Soviet work on sintering and combustion synthesis of porous nitinol from elemental powders. Li et al. ${ }^{6,7}$ have conducted extensive studies of high-temperature combustion synthesis of porous nitinol, in some cases using $\mathrm{TiH}_{2}$ as a pore-inducing agent. Porous nitinol has also been produced by hot isostatic pressing of elemental powders. ${ }^{8}$ The materials in these studies (whose relative densities were typically near 50\%) contained more-or-less randomly distributed void porosity that tended to increase local stress concentrations,

${ }^{\text {a)} E l e c t r o n i c ~ m a i l: ~ g r u m m o n @ e g r . m s u . e d u ~}$ with concomitant degradation of fracture strength and strainrecovery capability.

Our aim in the present experimental work is to produce a more carefully controlled, lower-density shape-memory/ superelastic material with a true open-cell foam structure, consisting of slender ligaments connecting an array of nodes. Because the principal deformation mode for such a material during crushing would involve bending and torsion (leading to buckling response and localized deformation of the ligaments), it is expected that efficient energy absorption, combined with damage-tolerant shape-recovery capability, can be realized.

For our first experiments, a powder-metallurgy method was used, in which a polymeric precursor foam was coated with a slurry of NiTi alloy powder. Gas-atomized 270-mesh $(\sim 50 \mu \mathrm{m}) \mathrm{Ti}_{49.1} \mathrm{Ni}_{50.9}$ powder (Special Metals Corp., New Hartford, NY), was combined with proprietary binders (Porvair Fuel Cell Technology Corp., Hendersonville, NC), and coated onto an open-cell polyurethane foam having an average cell size of 5 to $8.5 \mathrm{~mm}$. These specimens were then sintered in vacuum at various temperatures between 1422 and $1561 \mathrm{~K}$. The resulting foams were analyzed to determine interstitial contamination levels (using Leco CS200 and TC500 instruments), and transformation behavior (using a Perkin-Elmer Pyris-I differential scanning calorimeter). Both optical and electron micrographs were obtained, the latter using a Hitachi S-2500 scanning electron microscope (SEM).

The results of these exploratory trials varied widely as a function of sintering temperature and binder system, and only the highest temperature resulted in complete sintering. Processing at $1422 \mathrm{~K}$ produced very poor results, but when the sintering temperature was increased to $1477 \mathrm{~K}$, a distinct open-cell foam structure was created, as shown in Fig. 1. This foam had an approximate density of only $0.25 \mathrm{~g} / \mathrm{cm}^{3}$, or a relative density of $\sim 0.038$ compared to the $6.45 \mathrm{~g} / \mathrm{cm}^{3}$ nominal density of equiatomic NiTi. Sintering was still incomplete, however. Voids as large as $40 \mu \mathrm{m}$ were present in the microstructure of the struts and nodes, and the material 


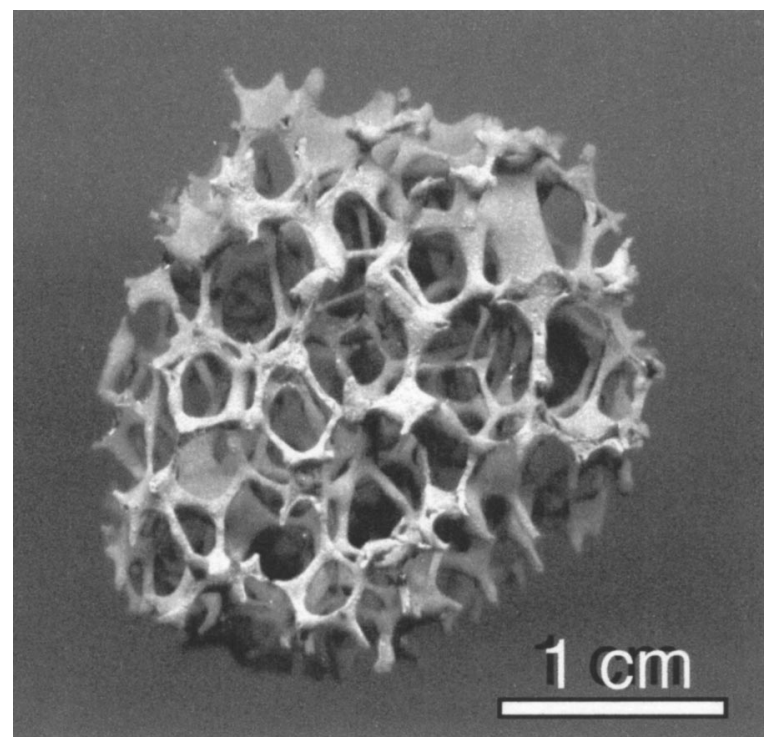

FIG. 1. Optical photograph of an open-cell NiTi foam sintered at $1477 \mathrm{~K}$.

was quite brittle. Increasing the sintering temperature further to $1533 \mathrm{~K}$ lead to a qualitative improvement in strength, but the resulting foam was still brittle due to incomplete sintering.

Only when the processing temperature was raised to $1561 \mathrm{~K}$ did sintering proceed to nominal completion. This represents a homologous temperature $\left(T / T_{m}\right)$ of 0.986 , so it is not surprising that incipient melting was found to have caused partial collapse of the foam structure during sintering. The result, however, was a reasonably well-formed foam (shown in Fig. 2) that displayed good strength and some evidence of room-temperature ductility. Although sintering was nominally complete (see Fig. 3), some void porosity was apparent.

The best overall results from the present series of experiments were obtained through the use of a two-stage approach in which the slurry-coating and sintering processes were carried out twice. After first processing a foam at $1479 \mathrm{~K}$, the sintered product was recoated with the powder slurry and

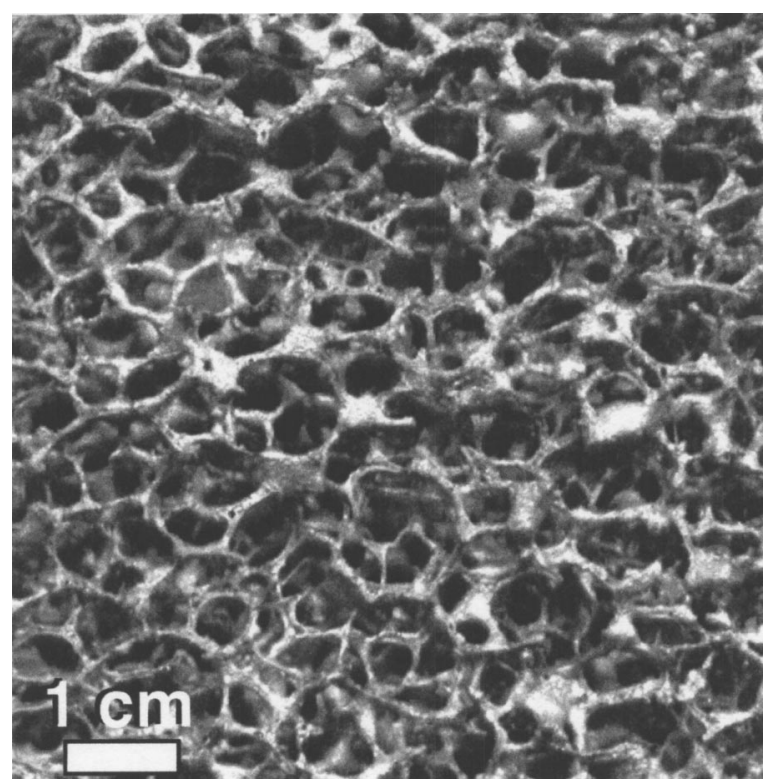

FIG. 2. Optical photograph of NiTi foam sintered at $1561 \mathrm{~K}$.

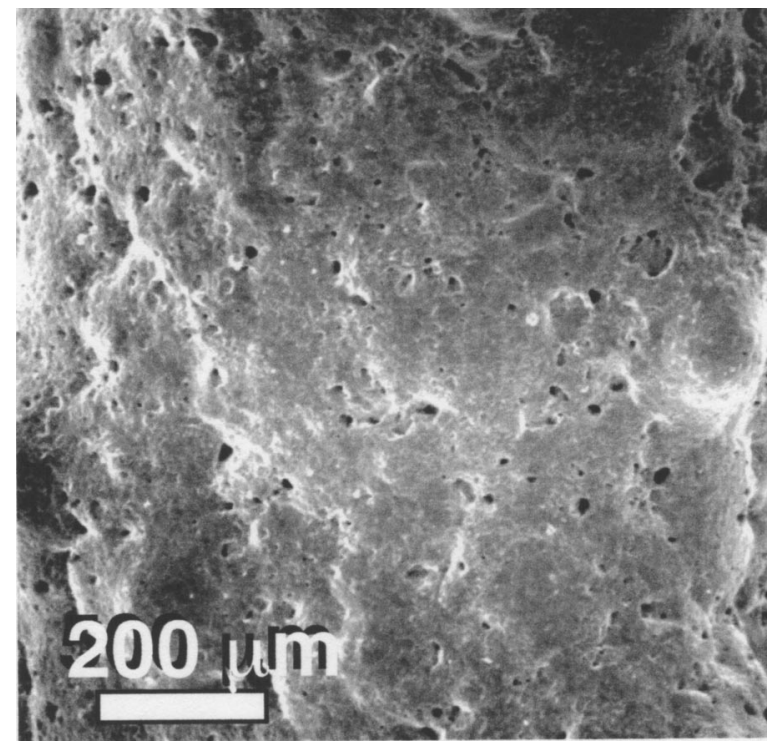

FIG. 3. Closeup SEM image showing complete sintering of the NiTi foam specimen shown in Fig. 2.

sintered a second time at $1561 \mathrm{~K}$. Although some local melting apparently occurred, the foam had qualitatively very high strength and exhibited moderate room-temperature ductility. (Detailed mechanical testing is in progress and quantitative results will be reported in a separate communication.) Microscopic examination of the surface revealed regions showing different degrees of sintering of the second powder layer, as can be seen in the pair of micrographs in Fig. 4. Figure 4(a) shows a region where probable localized melting left little trace of features of the original powder morphology. Figure 4(b) shows (at the same scale) a somewhat more typical surface region in which a high degree of sintering is evident, but a relatively large volume of free space remains around the coarsest of the sintered particles. It may thus be concluded that the $1561 \mathrm{~K}$ sintering temperature coincides closely with the solidus temperature for this alloy. We also note that the powders used in this work likely have composition variations connected with different rates of Ti volatilization during gas atomization, causing the smallest particles to become slightly more enriched in nickel. An increase in Ni content of as little as 0.1 at. \% can lower the solidus temperature by several tens of degrees, and may account for the variations observed in surface morphology.

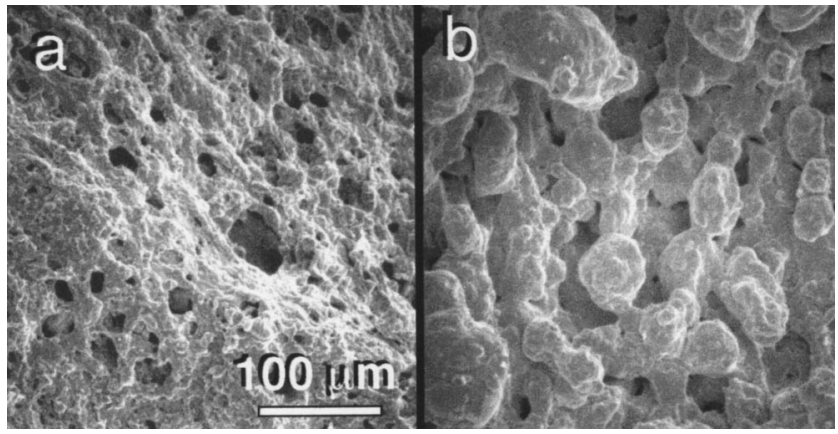

FIG. 4. Closeup SEM images showing surface details of a foam specimen processed by two-stage sintering (sintering of a second slurry applied to a first sintered layer). (a) Region showing evidence of melting. (b) More typical surface showing completely sintered powder with substantial free volume. 


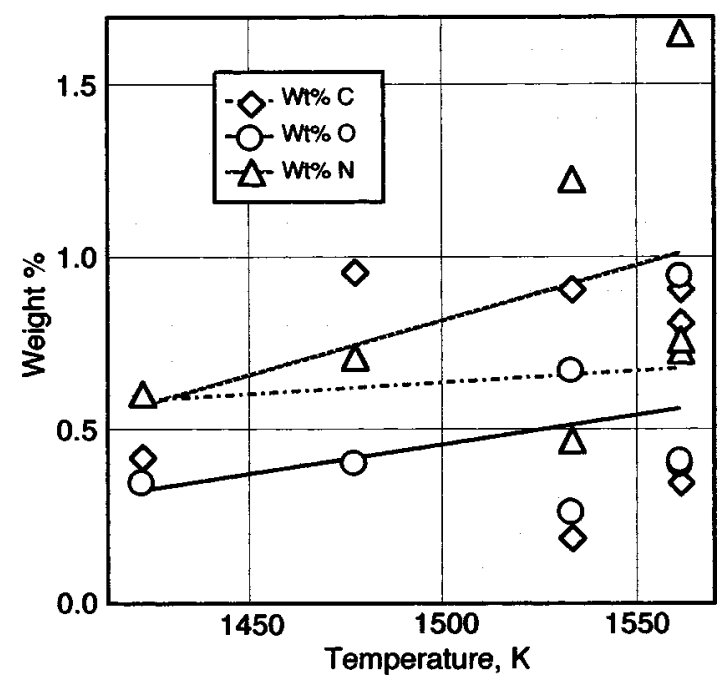

FIG. 5. Oxygen, nitrogen, and carbon interstitial contamination in the foams plotted as a function of sintering temperature.

Interstitial contamination was severe in all of the foams fabricated in this initial study, as may be appreciated from the data in Fig. 5, which shows oxygen, carbon, and nitrogen content of the films as a function of the sintering temperature. For processing at the highest temperatures, the nitrogen content in some cases exceeded $1 \mathrm{wt} \%$. In general, total interstitial contamination levels must be reduced by at least tenfold before good mechanical and transformational properties can be expected.

Nevertheless, clear evidence of displacive transformational behavior was obtained by differential scanning calorimetry (DSC). One such scan, made from material taken from the foam shown in Fig. 1, is shown in Fig. 6. Comparing these data to DSC scans from the raw, unsintered powder, it was observed that the transformational hysteresis was broadened, transformation temperatures were lowered, and transformational enthalpy was reduced. However, the fact that transformational response is found at all indicates that severe interstitial contamination may have affected only the outermost layers of the structure, and that the observed transformational enthalpies may have originated from the interior of the ligaments and nodes.

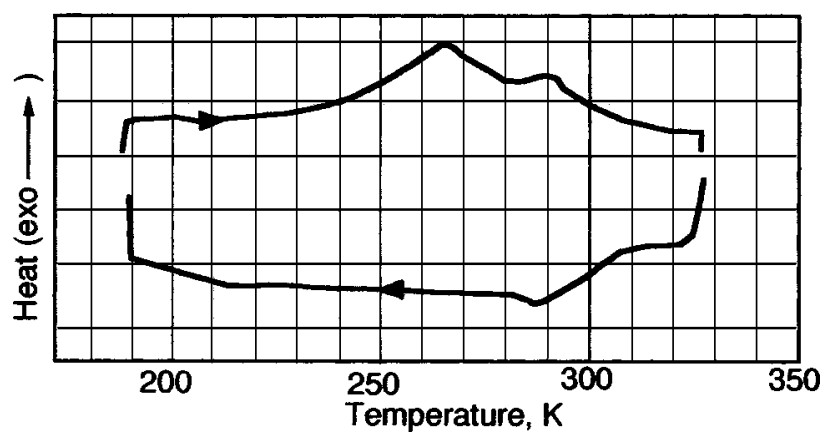

FIG. 6. DSC scan of the foam of Fig. 1 showing evidence of martensite transformation activity near 265 and $280 \mathrm{~K}$.

In summary, it has been shown that low-density opencell binary NiTi foams can be produced using a special powder-metallurgy method. Unlike previous techniques, the use of a polymeric precursor foam structure allows the final foam topology and cell spacing to be user selected. The approach can also achieve densities nearly an order of magnitude lower than previously reported methods for fabrication of simple porous nitinol. Although the foams produced thus far were severely contaminated with deleterious interstitial species, the foams exhibited definite martensitic transformation behavior and showed good mechanical integrity.

${ }^{1}$ Engineering Aspects of Shape Memory Alloys, edited by T. W. Duerig, K. N. Melton, D. Stockel, and C. M. Wayman (Butterworth-Heinemann, Washington, DC, 1990).

${ }^{2}$ Shape Memory Materials, edited by K. Otsuka and C. M. Wayman (Cambridge University Press, Cambridge UK, 1998).

${ }^{3}$ W. Ni, Y.-T. Cheng, and D. S. Grummon, Appl. Phys. Lett. 80, 3310 (2002).

${ }^{4}$ M. Ashby, A. Evans, N. Fleck, L. Gibson, J. Hutchinson, and H. Wadley, Metal Foams: A Design Guide, 1st ed. (Butterworth-Heinemann, Washington, DC, 2000).

${ }^{5}$ V. Itin, V. Gyunter, S. Shabalovskaya, and R. Sachdeva, Mater. Charact. 32, 179 (1994).

${ }^{6}$ B.-Y. Li, L.-J. Rong, V. Gjunter, and Y.-Y. Li, Z. Metallkd. 4, 291 (2000).

${ }^{7}$ B.-Y. Li, L.-J. Rong, Y.-Y. Li, and V. Gjunter, Metall. Mater. Trans. A 31, 1867 (2000).

${ }^{8}$ K. Thangaraj, Y. C. Chen, and K. Salama, Proceedings of the Adaptive Structures, and Material Systems Symposium, ASME International Mechanical Engineering Congress and Exposition, Orlando, FL, edited by J. Redmont and J. Main, Vol. 60, pp. 59-63. 